\title{
New and emerging trends in the treatment of atopic dermatitis
}

\author{
Christina M Gelbard' \\ Adelaide A Hebert ${ }^{1,2}$ \\ 'Departments of Dermatology; \\ ${ }^{2}$ Pediatrics, University \\ of Texas-Houston, Houston, \\ TX, USA
}

\begin{abstract}
Atopic dermatitis is a chronic, inflammatory skin condition that affects $10 \%$ to $20 \%$ of children and $1 \%$ to $3 \%$ of adults in the US. Symptoms often result in sleeplessness, psychological stress, poor self-esteem, anxiety, and poor school or work performance. The cost of atopic dermatitis is estimated to be US\$0.9 to 3.8 billion every year. Topical steroids are first-line treatment for atopic dermatitis, and recent advances in vehicle technologies have resulted in improved patient tolerability and compliance. Topical calcineurin inhibitors are also safe and effective topical treatments for atopic dermatitis, and provide an additional therapeutic option for patients with this disease. Systemic immunomodulators are used in the treatment of severe refractory disease. Cyclosporine, methotrexate, azathioprine, mycophenolate mofetil, and interferon gamma have been used in the management of severe atopic dermatitis. This review highlights the current and emerging trends in the treatment of atopic dermatitis.

Keywords: atopic dermatitis, topical corticosteroids, calcineurin inhibitors, methotrexate, cyclosporine, mycophenolate mofetil, IFN- $\gamma$
\end{abstract}

\section{Introduction}

Atopic dermatitis is a chronic, inflammatory skin condition that affects $10 \%$ to $20 \%$ of children and $1 \%$ to $3 \%$ of adults in the US (Lipozencic and Wolf 2007). Symptoms include erythema, scaling and pruritus in affected areas, and often result in sleeplessness, psychological stress, poor self-esteem, anxiety, and poor school or work preformance. The cost of atopic dermatitis is estimated to be US\$0.9 to 3.8 billion every year, with direct medical costs and out-of-pocket expenses averaging US\$609/patient/year (Munzenberger and Montejo 2007). Topical steroids have been the mainstay of treatment for atopic dermatitis in both children and adults for over 50 years. Recent advances in vehicle technologies have resulted in the development of novel gel and foam formations of several topical steroids, and have improved patient tolerability and compliance. Although the efficacy of topical steroids is wellestablished, use may be limited by potential side effects and many patients with more severe disease require additional therapy. Topical calcineurin inhibitors have proven to be safe and effective in the treatment of atopic dermatitis, providing an additional therapeutic option for patients with this disease. In addition to advances in topical treatments for atopic dermatitis, systemic immunomodulators are becoming more prevalent in the treatment of severe, refractory disease. Cyclosporine, methotrexate, azathioprine, mycophenolate mofetil, and interferon gamma (IFN- $\gamma$ ) have been used in the management of severe atopic dermatitis. The aim of this review is to present the current and emerging trends in the treatment of atopic dermatitis.

\section{Topical steroids: new formulations}

The use of topical corticosteroids in dermatology began only 50 years ago, yet these medications are now considered first-line therapy for adult and pediatric patients 
with atopic dermatitis (Stahn et al 2007). Topical steroids have immunosuppressive, anti-inflammatory, antiproliferative, and vasoconstrictive effects that have been shown to reduce itching, improve the appearance of skin, and improve quality of life. Until recently, only creams, ointments, and lotions were available, but now new hydrogel and foam formulations have been developed. These advances in vehicle technology provide additional therapeutic options for patients with atopic dermatitis, and may enhance patient compliance and acceptability. Side effects associated with topical steroid use include cutaneous atrophy, striae, telangiectasias, contact dermatitis, Cushing's syndrome, growth retardation, and suppression of the hypothalmamicpituitary-adrenal axis (Eichenfield et al 2007; Wolverton 2007). These potential side effects can be concerning to both patients and physicians and may result in decreased prescribing and compliance.

\section{Topical calcineurin inhibitors}

Topical tacrolimus and pimecrolimus are calcineurin inhibitors that were developed for the treatment of atopic dermatitis. Inhibition of calcineurin results in decreased transcription of IL-2, with resulting decreases in T-cell activation and cytokine production. Topical calcineurin inhibitors (TCIs) cause depletion of inflammatory dendritic cells in atopic dermatitis lesions, decrease histamine release from mast cells and basophils, and prevent the T-cell proliferation that results from Staphylococcus aureus superantigens (Wolverton 2007). One of the greatest advantages of these newer medications is the absence of many of the side effects associated with topical corticosteroids, including cutaneous atrophy, striae, telangiectasias, and hypothalamicpituitary-adrenal (HPA) axis suppression. TCIs have been shown to be safe and effective in numerous clinical trials, and provide additional therapeutic options for the treatment of atopic dermatitis.

Currently, Food and Drug Administration (FDA) guidelines for the use of TCIs recommend that these medications should not be used in children younger than 2 years of age or in immunocompromised patients. TCIs are indicated as short-term or non-continuous, long-term treatment of atopic dermatitis in patients who have not responded to other topical treatments, or in whom these other treatments are not recommended. Pimecrolimus 1\% cream is FDA-approved for the treatment of mild to moderate atopic dermatitis in patients 2 years of age or older. Tacrolimus $0.1 \%$ ointment is approved for the treatment of moderate to severe atopic dermatitis in patients 16 years of age or older, and tacrolimus $0.03 \%$ ointment is approved for use in patients 2 years of age or older (Paller et al 2005; Munzenberger and Montejo 2007; Wolverton 2007).

A comparative study of tacrolimus $0.1 \%$ ointment demonstrated efficacy comparable to the midpotency corticosteroid hydrocortisone butyrate $0.1 \%$ (Reitamo et al 2002a). A study of pediatric patients showed superior efficacy of $0.03 \%$ and $0.1 \%$ tacrolimus ointment when compared to hydrocortisone acetate 1\% (Reitamo et al 2002b).

Multiple clinical trials evaluating pimecrolimus cream $1 \%$ for the treatment of atopic dermatitis demonstrated superior efficacy when compared with vehicle creams, as well as reduced frequency of flares, and reduced need for topical corticosteroids (Lebwohl and Gower 2006).

Finally, 3 multi-center, randomized, investigator-blinded studies involving a total of 1065 adult and pediatric patients with mild to very severe atopic dermatitis compared the efficacy and safety of tacrolimus ointment to pimecrolimus cream. Tacrolimus ointment was found to be significantly more effective than pimecrolimus cream in adults and children with moderate to very severe disease. In pediatric patients with mild disease, the improvements in the tacrolimus group were significantly greater at week one and trended towards continued advantage at the end of the study. Patients treated with tacrolimus also showed faster onset of action, and greater improvement in itch scores, percentage of body surface area affected and Investigator Global AD Assessment scores. There was no significant difference in adverse events between the two treatment groups. The number of local application site reactions experienced on day one was greater in adults treated with tacrolimus, but the incidence on all subsequent days was equal in both groups. More patients in the pimecrolimus group withdrew from the studies due to lack of efficacy (Paller et al 2005).

The most common adverse effects associated with the use of TCIs are local application site reactions. These include skin burning, stinging and pruritus. Unlike topical corticosteroids, TCIs have not been found to cause HPA axis suppression, skin atrophy, striae, or telangiectasias, and are safe to use even on the thin skin of the face and neck.

A black box warning and patient medication guide were added to TCI labels in January 2006 due to safety concerns raised by the FDA (Lebwohl and Gower 2006). These concerns were related to the immunosuppression and increased incidence of lymphoproliferative disease associated with systemically-administered calcineurin inhibitors in transplant patients, and to studies in animal models involving high doses of orally-administered drug. Although rare cases 
of lymphoma and skin malignancies have been reported in post-marketing surveillance studies, no causal relationship has been established between these cases and the use of TCIs, and the reported lymphoma cases were no greater than the expected rate in the general population (Lebwohl and Gower 2006; Munzenberger and Montejo 2007). Numerous pharmacokinetic studies have shown that the administration of topical pimecrolimus or tacrolimus results in negligible amounts of drug absorption into the systemic circulation (Munzenberger and Montejo 2007). After careful review of safety data, The American Academy of Dermatology, the American College of Allergy, Asthma and Immunology and the American Academy of Allergy, Asthma and Immunology released statements that data did not suggest increased malignancy risk with the use of TCIs and that they did not support the black-box warning (Lebwohl and Gower 2006; Munzenberger and Montejo 2007).

\section{Cyclosporine}

Cyclosporine A (CsA) was isolated from the soil fungus Tolypocladium inflatum in 1970. This medication is a potent immunosuppressant that inhibits cell-mediated immunity, and was approved in the US in 1983 for the prophylaxis of organ transplant rejection (Lee et al 2004; Wolverton 2007). Cyclosporine is now approved in the US for the treatment of psoriasis, and is licensed in Europe for the short-term treatment of severe atopic dermatitis in adults (Harper et al 2000). In the US, cyclosporine is used off-label for the shortterm treatment of severe atopic dermatitis in both children and adults. Cylosponine shares a common mechanism of action with the TCIs. This medication inhibits calcineurnin, resulting in decreased production of IL-2 and other inflammatory cytokines and preventing the activation and proliferation of T cells. Due to its high molecular weight, cyclosporine is not effective topically and must be administered orally.

A large meta-analysis of 15 European studies evaluated the use of cyclosporine in the treatment of severe atopic dermatitis in over 600 children and adults. Treatment duration ranged from 6 weeks to 12 months. All studies showed a mean decrease in the severity of atopic dermatitis. Twelve of the studies were pooled, and the results demonstrated doserelated improvements in atopic dermatitis with cyclosporine treatment. Two weeks into treatment, patients receiving low-dose therapy $(\leq 3 \mathrm{mg} / \mathrm{kg}$ ) showed a mean decrease in disease severity of $22 \%$, and patients on high dose therapy ( $\geq 4 \mathrm{mg} / \mathrm{kg}$ ) showed a $40 \%$ decrease in disease severity. After 6 to 8 weeks, relative effectiveness was $55 \%$. Three of the included studies assessed health-related quality of life and all three found significant improvements with cyclosporine treatment. Adverse events included reversible increases in creatinine, hypertension, infections, gastrointestinal symptoms, headache and paresthesias (Schmitt et al 2007).

A retrospective review of 15 Chinese patients ages 1 to 58 years with severe atopic dermatitis treated with oral cyclosporine demonstrated rapid improvement in disease severity 2 weeks after initiating treatment. At the end of treatment, $73 \%$ of the patients showed improvement in their disease; however, relapses were noted in all patients within 3 months of cessation of cyclosporine. Worsening blood pressure occurred in 1 patient who had borderline hypertension prior to cyclosporine treatment; return to baseline occurred after cessation of cyclosporine (Lee et al 2004).

A retrospective study of 73 adult patients from the Netherlands evaluated the long-term safety and efficacy of cyclosporine A for the treatment of atopic dermatitis. Patients received treatment for an average of 1.3 years, with doses ranging from 2.5 to $5 \mathrm{mg} / \mathrm{kg} /$ day. Successful treatment (50\%-100\% improvement) was achieved in 77\%, moderate $(<50 \%)$ improvement in $16 \%$, and no improvement in $7 \%$ of patients. The authors note that most patients demonstrated rapid improvement within the first few weeks of treatment. Serum creatinine levels rose $>30 \%$ from baseline in $9.6 \%$ of patients, and hypertension developed in $15.1 \%$ of patients. Other side effects reported included paresthesias, hypertrichosis, headaches, fatigue, nausea, gingival hyperplasia, muscle cramps, folliculitis, and tremors. Upon discontinuation of cyclosporine, $45 \%$ of the patients experienced remission of their atopic dermatitis for at least 3 months, and $55 \%$ experienced relapse. Of note, $8 \%$ of patients who relapsed developed rebound disease that was more severe than baseline (Hijnen et al 2007).

A prospective, randomized trial of 40 British children with atopic dermatitis compared the efficacy, safety, tolerability and quality of life of short-course and continuous treatment with cyclosporine. Children ages 2 to 16 years with severe atopic dermatitis were treated with either multiple short courses of 12 weeks each of cyclosporine $5 \mathrm{mg} / \mathrm{kg}$ or with continuous therapy for one year. Improvements in severity, area of involvement, irritability and sleep disturbance were statistically significant in both groups from baseline to weeks 8,12 , and month 12 . Although no significant differences were seen between the groups at any particular time point, improvements fluctuated in the short course arm as a result of medication tapering and cessation. No patients showed clinically significant changes in serum creatinine or blood pressure, and tolerability was considered 
good or very good in at least $80 \%$ of the patients. Although the mean cumulative dose of cyclosporine was greater in continuous arm than in the short-course arm (1068 and $836 \mathrm{mg} / \mathrm{kg}$, respectively), the patients in the continuous arm demonstrated more consistent disease control (Harper et al 2000).

\section{Methotrexate}

Methotrexate is a folic acid antagonist that is approved for the treatment of psoriasis, rheumatoid arthritis, and mycosis fungoides (Wolverton 2007). Randomized controlled trials are lacking, but several small trials reported clinical improvement of moderate to severe atopic dermatitis in patients treated with methotraxate. A prospective trial of 12 patients evaluated the use of methotrexate for the treatment of moderate to severe atopic dermatitis. The average improvement in disease activity was 50\% from baseline, and there were significant improvements in quality of life, affected body surface area, and loss of sleep and itch scores (Weatherhead et al 2007). Although the use of methotrexate in atopic dermatitis is off label, its anti-inflammatory effects make it a useful second-line agent for the treatment of atopic dermatitis. Side effects include nausea and elevated liver enzymes, and patients occasionally develop pancytopenia or hepatic or pulmonary toxicity (Goujon et al 2006; Weatherhead et al 2007; Wolverton 2007). Patients should be monitored closely when therapy is intiated, with complete blood count, liver enzymes, and creatinine levels 1 to 2 weeks after initiation of therapy and after any changes in dosing. Once long-term dosing levels have been established, repeat testing should be obtained every 3 to 4 months (Wolverton 2007).

\section{Azathioprine}

Azathioprine was initially developed for the prevention of graft rejection in organ transplant patients. Additionally, azathiaprine's immunosuppressive properties make it a useful treatment for a number of diseases, including systemic lupus erythematosis, dermatomyositis, pemphigus vulgaris, and atopic dermatitis (Meggitt and Reynolds 2001). Azathioprine is converted into 6-mercaptopurine, a purine analog that produces metabolites that incorporate into DNA. Although data on the use of azathioprine for atopic dermatitis are limited, there are several studies that support its use as a second-line treatment for severe, refractory disease. A review of 8 studies involving a total of 128 adult and pediatric patients treated with azathioprine documented overall improvement in atopic dermatitis symptoms in 7 of the 8 studies (Meggitt and Reynolds 2001). In addition, a double-blind, randomized, placebo-controlled crossover study evaluated the safety and efficacy of azathioprine $2.5 \mathrm{mg} / \mathrm{kg} /$ day for 12 weeks in adult patients with severe atopic dermatitis. Subjects were evaluated using the SASSAD (Six Area Six Sign Atopic Dermatitis) sign score and demonstrated $26 \%$ reduction during azathioprine treatment, but only a 3\% reduction with placebo. Pruritus, sleep disturbance, and disruption of work/daytime activity showed significant improvements during azathioprine treatment but not with placebo treatment. While receiving azathioprine treatment, 14 subjects reported gastrointestinal side effects, 8 demonstrated abnormalities in their liver enzymes, and 2 became leukopenic (Berth-Jones et al 2002).

The most common serious side effects of azathioprine are dose-dependant hepatotoxicity and myelotoxicity. Myelotoxicity has been shown to be related to activity of thiopurine methyltransferase (TPMT), an enzyme necessary for the metabolism of azathioprine. Approximately $10 \%$ of the population are heterozygous at the TPMT locus and demonstrate intermediate TPMT activity, putting them at increased risk for myelosupression when treated with azathoprine. Homozygous deficiency of TPMT is found in $0.33 \%$ of the population, and these individuals are likely to show profound myelosuppression when treated with azathioprine (Meggitt and Reynolds 2001). A double-blind, placebo-controlled trial evaluated the safety and efficacy of azathioprine dosed by TPMT activity in moderate to severe atopic dermatitis. Patients with normal TPMT activity received $2.5 \mathrm{mg} / \mathrm{kg} / \mathrm{day}$, while those with heterozygous range TPMT activity received $1 \mathrm{mg} / \mathrm{kg}$.day. At week 12, there was a 37\% improvement in mean disease activity in the azathioprine group compared with $20 \%$ in the placebo group. Although response to the drug varied widely among participants, subjects with heterozygous TPMT activity responded to the medication in similar proportions to the participants with normal TPMT activity, and did not develop myelotoxicity (Meggitt et al 2006).

Concerns also exist that long-term use of this medication may increase the risk of certain malignancies. These concerns stem from the increased rate of squamous cell skin cancers and non-Hodgkin's lymphoma in organ transplant patients who have received long-term immunosuppressive therapy that included azathioprine. However, studies of patients taking short- or medium-term courses of azathioprine do not demonstrate this increased risk of carcinogenicity (Meggitt and Reynolds 2001).

\section{Mycophenolate mofetil}

Mycophenolate mofetil (MMF) has been used recently in the treatment of adult and pediatric patients with moderate 
to severe refractory atopic dermatitis. Although randomized controlled trials have not yet been done, several studies have demonstrated symptom improvement in patients treated with this drug (Heller et al 2007; Murray and Cohen 2007).

MMF is a selective reversible inhibitor of inosine monophosphate dehydrogenase, an enzyme necessary for the de novo synthesis of purines. Lymphocytes lack the salvage pathway present in other inflammatory cell types, and are therefore dependent on the de novo synthesis pathway. Inosine monophosphate dehydrogenase inhibition results in selective suppression of lymphocytes.

A retrospective chart review examined 20 adult patients with moderate to severe atopic dermatitis treated with mycophenolate mofetil at starting doses of $500 \mathrm{mg}$ to $1 \mathrm{~g}$ twice daily. Seventeen of the 20 patients showed improvement in disease severity after 2 to 4 weeks and were maintained on treatment. Ten patients eventually achieved complete clearance of atopic dermatitis and were tapered off of MMF. Although MMF was generally well tolerated, side effects included mild headaches, gastrointestinal complaints and fatigue. Four patients developed herpes zoster, 1 developed herpes simplex, and 2 developed cutaneous infections with Staphylococcus aureus (Murray and Cohen 2007).

A retrospective study of 14 pediatric patients with severe atopic dermatitis demonstrated that MMF was both a safe and effective treatment option. Over half of the patients achieved complete or almost complete clearance, and only one failed to respond. The authors initiated dosing at 40 to $50 \mathrm{mg} / \mathrm{kg}$ daily in younger children and 30 to $40 \mathrm{mg} / \mathrm{kg}$ daily in adolescents. They noted that initial responses typically occurred within 2 months, with maximal effect attained after 2 to 3 months. They reported no infectious complications in their cohort (Heller et al 2007).

\section{IFN- $\gamma$}

Interferon gamma (IFN- $\gamma)$ is a cytokine that exerts anti-inflammatory effects by inhibiting $\operatorname{IgE}$ synthesis and $\mathrm{T}$ cell proliferation (Meagher et al 2002). Treatment with IFN- $\gamma$ has been shown to decrease blood eosinophil counts in patients with atopic dermatitis, and has been shown to improve atopic dermatitis symptoms in patients with severe disease (Jang et al 2000). A controlled trial of 51 patients with severe, recalcitrant atopic dermatitis compared low-dose $\left(0.5 \times 10^{6} \mathrm{IU} / \mathrm{m}^{2}\right) \mathrm{IFN}-\gamma$, high-dose $\left(1.5 \times 10^{6} \mathrm{IU} / \mathrm{m}^{2}\right) \mathrm{IFN}-\gamma$, and placebo treatment 3 times per week over 12 weeks. Both groups treated with IFN- $\gamma$ showed significant reduction in disease severity. The high-dose group showed more rapid and effective treatment outcome at week 6 , but improvement in both groups remained stable after week 8 , leading the authors to suggest a regimen of initial high-dose therapy, followed by maintenance therapy at the lower dosage. Potential side effects associated with IFN- $\gamma$ therapy include transient fever, myalgias, respiratory difficulties, and elevation of $\mathrm{LDH}$ or transaminases (Jang et al 2000).

\section{Conclusion}

Atopic dermatitis is a chronic inflammatory skin disease that affects both children and adults and causes significant physical, psychological, social, and emotional stress for patients and their families. Although topical steroids have been the first-line treatment for atopic dermatitis for the past half century, advances in our understanding of disease pathogenesis and new developments in immunosuppressive medications have expanded the therapeutic options available to treat this disease. The topical medications available for atopic dermatitis have expanded to incorporate new vehicle technologies as well as steroid-sparing topical calcineurin inhibitors. The use of systemic immunosuppressants, including cyclosporine, methotrexate, azathioprine, mycophenilate mofetil, and IFN- $\gamma$ has become more common, particularly for severe, refractory disease.

\section{Disclosures}

Dr. Adelaide Herbert has been a researcher, lecturer and consultant for Astellas Pharma and Novartis.

\section{References}

Berth-Jones J, Takwale A, Tan E, et al. 2002. Azathioprine in severe adult atopic dermatitis: a double-blind, placebo-controlled, crossover trial. BrJ Dermatol, 147:324-30.

Eichenfield LF, Basu S, Calvarese B, et al. 2007. Effect of desonide hydrogel $0.05 \%$ on the hypothalamic-pituitary-adrenal axis in pediatric subjects with moderate to severe atopic dermatitis. Pediatr Dermatol, 24:289-95.

Goujon C, Berard F, Dahel K, et al. 2006. Methotrexate for the treatment of adult atopic dermatitis. Eur J Dermatol, 16:155-8.

Harper JI, Ahmed I, Barclay G, et al. 2000. Cyclosporin for severe childhood atopic dermatitis: short course versus continuous therapy. Br J Dermatol, 142:52-8.

Heller M, Shin HT, Orlow SJ, et al. 2007. Mycophenolate mofetil for severe childhood atopic dermatitis: experience in 14 patients. Br J Dermatol, 157:127-32.

Hijnen DJ, Ten Berge O, Timmer-De Mik L, et al. 2007. Efficacy and safety of long-term treatment with cyclosporin A for atopic dermatitis. $J$ Eur Acad Dermatol Venereol, 2185-9.

Jang IG, Yang JK, Lee HJ, et al. 2000. Clinical improvement and immunohistochemical findings in severe atopic dermatitis treated with interferon gamma. J Am Acad Dermatol, 42:1033-40.

Lebwohl M, Gower T. 2006. A safety assessment of topical calcineurin inhibitors in the treatment of atopic dermatitis. Med Gen Med, 8:8.

Lee, SS, Tan, AW, Giam, YC. 2004. Cyclosporin in the treatment of severe atopic dermatitis: a retrospective study. Ann Acad Med Singapore, 33:311-3.

Lipozencic J, Wolf R. 2007. Atopic dermatitis: an update and review of the literature. Dermatol Clin, 25:05-12, x. 
Meagher LJ, Wines NY, Cooper AJ. 2002. Atopic dermatitis: review of immunopathogenesis and advances in immunosuppressive therapy. Australas J Dermatol, 43:47-54.

Meggitt SJ, Gray JC, Reynolds NJ. 2006. Azathioprine dosed by thiopurine methyltransferase activity for moderate-to-severe atopic eczema: a double-blind, randomised controlled trial. Lancet, 367:39-46.

Meggitt SJ, Reynolds NJ. 2001. Azathioprine for atopic dermatitis. Clin Exp Dermatol, 26:69-75.

Munzenberger PJ, Montejo JM. 2007. Safety of topical calcineurin inhibitors for the treatment of atopic dermatitis. Pharmacotherapy, 27:020-8

Murray ML, Cohen JB. 2007. Mycophenolate mofetil therapy for moderate to severe atopic dermatitis. Clin Exp Dermatol, 32:3-7.

Paller AS, Lebwohl M, Fleischer AB Jr, et al. 2005. Tacrolimus ointment is more effective than pimecrolimus cream with a similar safety profile in the treatment of atopic dermatitis: results from 3 randomized, comparative studies. J Am Acad Dermatol, 52:810-22.
Reitamo S, Rustin M, Ruzicka T, et al. 2002a. Efficacy and safety of tacrolimus ointment compared with that of hydrocortisone butyrate ointment in adult patients with atopic dermatitis. J Allergy Clin Immunol, 109:547-55.

Reitamo S, van Leent EJ, Ho V, et al. 2002b. Efficacy and safety of tacrolimus ointment compared with that of hydrocortisone acetate ointment in children with atopic dermatitis. J Allergy Clin Immunol, 109:539-46.

Schmitt J, Schmitt N, Meurer M. 2007. Cyclosporin in the treatment of patients with atopic eczema - a systematic review and meta-analysis. J Eur Acad Dermatol Venereol, 21:606-19.

Stahn C, Lowenberg M, Hommes DW, et al. 2007. Molecular mechanisms of glucocorticoid action and selective glucocorticoid receptor agonists. Mol Cell Endocrinol, 275:71-8.

Weatherhead SC, Wahie S, Reynolds NJ, et al. 2007. An open-label, dose-ranging study of methotrexate for moderate-to-severe adult atopic eczema. Br J Dermatol, 156:346-51.

Wolverton S. 2007. Comprehensive dermatologic drug therapy. 2nd ed. Philadelphia: Elsevier. 